Revista de Educação e Pesquisa em Contabilidade

Journal of Education and Research in Accounting

Revista de Educación e Investigatión en Contabilidad
REPeC, Brasília, v. 6, n. 3, informações editoriais, p. 343-345, jul./set. 2012

Disponível online em www.repec.org.br

ISSN 1981-8610

\title{
Informações Editoriais
}

Periódico Trimestral, digital e gratuito, do Conselho Federal de Contabilidade (CFC), Fundação Brasileira de Contabilidade (FBC) e Academia Brasileira de Ciências Contábeis (ABRACICON).

Data de publicação: 14 de setembro de 2012

\section{EQUIPE EDITORIAL}

\section{CORPO DIRETIVO}

Juarez Domingues Carneiro, Presidente do Conselho Federal de Contabilidade (CFC), Brasil

José Martonio Alves Coelho, Presidente da Fundação Brasileira de Contabilidade (FBC), Brasil

Maria Clara Cavalcante Bugarim, Presidente da Academia Brasileira de Ciências Contábeis (ABRACICON), Brasil

\section{COMITÊ DE POLÍTICA EDITORIAL}

Antonio Carlos Dias Coelho, Doutor, Universidade Federal do Ceará (UFC), CE, Brasil

Edgard B. Cornachione Jr., Ph.D., Universidade de São Paulo (USP), SP, Brasil

Ernani Ott, Doutor, Universidade do Vale do Rio dos Sinos (Unisinos), RS, Brasil

Ilse Maria Beuren, Doutora, Universidade Regional de Blumenau (FURB), SC, Brasil

Jorge Katsumi Niyama, Doutor, Universidade de Brasília (UNB), DF, Brasil

Jacqueline Veneroso Alves da Cunha, Doutora, Universidade Federal de Minas Gerais (UFMG), MG, Brasil Valcemiro Nossa, Doutor, Fundação Instituto Capixaba de Pesquisas em Contabilidade, Economia e Finanças (Fucape),ES, Brasil

\section{EDITOR}

Valcemiro Nossa, Doutor, Fundação Instituto Capixaba de Pesquisas em Contabilidade, Economia e Finanças (Fucape),ES, Brasil

\section{EDITORES ADJUNTOS}

Carlos Renato Theóphilo, Doutor, Universidade Estadual de Montes Claros (Unimontes), MG, Brasil Flávia Zóboli Dalmácio, Doutora, Universidade de São paulo (USP), SP, Brasil

Romualdo Douglas Colauto, Doutor, Universidade Federal do Paraná (UFPR), PR, Brasil

Vera Maria Rodrigues Ponte, Doutora, Universidade Federal do Ceará (UFC), CE, Brasil

Copyright (C 2012 REPEC. Todos os direitos, até mesmo de tradução, são reservados. É permitido citar parte de artigos sem autorização prévia, desde que seja identificada a fonte. 


\section{CORPO EDITORIAL CIENTÍFICO}

Adriano Rodrigues, Doutor, Universidade Federal do Rio de Janeiro (UFRJ), RJ, Brasil Alfredo Sarlo Neto, Doutor, Universidade Federal do Espírito Santo (UFES), ES, Brasil Aneide Oliveira Araujo, Doutora, Universidade Federal do Rio Grande do Norte (UFRN), RN, Brasil Cláudio Parisi, Doutor, Fundação Escola de Comércio Álvares Penteado (FECAP), SP, Brasil Edílson Paulo, Doutor, Universidade Federal da Paraíba (UFPB), PB, Brasil

Eduardo Schiehll, PhD, HEC Montreal, Canada

Fernando Caio Galdi, Doutor, Fundacao Instituto Capixaba de Pesquisas em Contabilidade, Economia e Finanças (Fucape),ES, Brasil

Jeronymo José Libonati, Doutor, Universidade Federal de Pernambuco (UFPE), PE, Brasil

Jorge Eduardo Scarpin, Doutor, Universidade Regional de Blumenau (FURB), SC, Brasil

José Alonso Borba, Doutor, Universidade Federal de Santa Catarina (UFSC), SC, Brasil

Lauro Brito de Almeida, Doutor, Universidade Federal do Paraná (UFPR), PR, Brasil

Leandro Cañibano, Doutor, Universidad Autonoma de Madrid, Espanha

Lino Martins da Silva, Doutor, Universidade Estadual do Rio de Janeiro (UERJ), RJ, Brasil

Luis Eduardo Afonso, Doutor, Universidade de São paulo (USP), SP, Brasil

Luís Lima Santos, Doutor, Instituto Politécnico de Leiria (IPL), Portugal

Maisa de Souza Ribeiro, Doutora, Universidade de São Paulo de Ribeirão Preto (USP-RP), SP, Brasil

Márcia Martins Mendes De Luca, Doutora, Universidade Federal do Ceará (UFC), CE, Brasil

Marcos Antonio Souza, Doutor, Universidade do Vale do Rio dos Sinos (Unisinos), RS, Brasil

Milanez Silva de Souza, Doutor, Universidade Federal do Amazonas (UFAM), AM, Brasil

Sonia Maria da Silva Gomes, Doutora, Universidade Federal da Bahia (UFBA), BA, Brasil

\section{AVALIADORES AD HOC}

Lista publicada anualmente no último número da Revista

\section{REVISÃO DE LÍNGUA PORTUGUESA}

Maria do Carmo Nóbrega, Conselho Federal de Contabilidade (CFC), Brasil

\section{REVISÃO DE LÍNGUA INGLESA}

Milton Coelho, Positive Idiomas

Clarissa Brocca, Positive Idiomas

\section{REVISÃO DA LÍNGUA ESPANHOLA}

Juan Canellas, Positive Idiomas

\section{REVISÃO DE NORMALIZAÇÃO}

Maria do Carmo Nóbrega, Conselho Federal de Contabilidade (CFC), Brasil

\section{PREPARAÇÃO/REVISÃO DE METADADOS}

Geraldo Augusto Morais Figueiredo, Conselho Federal de Contabilidade (CFC), Brasil

\section{DIAGRAMAÇÃO}

Thiago Luis Gomes, Conselho Federal de Contabilidade (CFC), Brasil 


\section{BIBLIOTECÁRIA}

Lúcia Helena Alves de Figueiredo, Conselho Federal de Contabilidade (CFC), Brasil

\section{ASSISTENTE EDITORIAL}

Rosangela Bekman dos Santos, Conselho Federal de Contabilidade (CFC), Brasil

\section{(C) CFC - Conselho Federal de Contabilidade}

Esta revista oferece acesso livre imediato ao seu conteúdo, pela internet no sistema SEER, disponibilizando gratuitamente o conhecimento científico ao público com vistas à sua democratização.

A REPeC está utilizando o Sistema Eletrônico de Editoração de Revistas (SEER) como meio de gerenciamento do periódico.

O Sistema Eletrônico de Editoração (SEER) foi traduzido e adaptado do Open Journal Systems (OJS) pelo Instituto Brasileiro de Informação em Ciência e Tecnologia (IBICT). Esse software livre, desenvolvido pela Universidade British Columbia do Canadá, tem como objetivo dar assistência na edição de periódicos científicos em cada uma das etapas do processo, desde a submissão e avaliação dos consultores até a publicação on-line e sua indexação.

Disponível em: http://www.repec.org.br

REPeC: Revista de Educação e Pesquisa em Contabilidade [recurso eletrônico] / Conselho Federal de Contabilidade. - V.1, n.1 (dez. 2006 /mar. 2007)- . -- Brasília: CFC, 2007-.

Trimestral

ISSN: 1981-8610

Modo de acesso: http://www.repec.org.br

1. Ciências Contábeis. 2. Contabilidade I. Conselho Federal de Contabilidade II. Título.

Ficha Catalográfica elaborada pela Bibliotecária Lúcia Helena Alves de Figueiredo CRB 1/1.401

\section{ENDEREÇO:}

Conselho Federal de Contabilidade (CFC)

SAS quadra 5 , bloco $\mathrm{J}$

Edifício CFC

70.070-920 Brasília - DF

E-mail: repec@cfc.org.br 